\title{
Neck Pain and Functioning in Daily Activities Associated with Smartphone Usage
}

Hae-jung Lee

Department of Physical Therapy, College of Health \& Welfare, Silla University, Busan, Korea

Purpose: The aim of the study was to investigate neck posture, range of motion, muscle endurance and self-report of pain and disability in smartphone users.

Methods: Seventy-eight university student volunteers, aged between 18 and 30 years (mean age 23.2), were assessed for: a head-neck posture by measuring cranial vertical angle, neck range of motions using cervical range of motion device, and a deep neck flexor endurance using a stabilizer. Finally, subjects were asked about their neck pain and completed disability questionnaires, ie, Short Form McGill Pain Questionnaire, Neck Disability Index, and World Health Organization Disability Assessment Schedule 2.0.

Results: Thirty-eight subjects experienced recurrent neck pain with/without upper limb pain (neck pain group) and 40 reported no current neck pain with/without upper limb pain (no neck pain group). Differences were found between groups on pain and disability questionnaires. Subjects with neck pain had significantly higher disability scores than those of no neck pain group. However, there were no differences observed between groups in a head-neck posture, neck range of motions, and deep neck muscle endurance time. The smartphone usage time was negatively correlated with neck pain intensity and disability score whereas it had positive relationship with flexibility and posture.

Conclusion: Group differences were observed as lower capacity not only for neck specific daily activities but for general functioning in daily routine when the neck pain and no neck pain groups were compared. Therefore, functioning in daily activities should be investigated as prevention for further developing neck pain in smartphone users.

Keywords: Neck pain, Functioning, Smartphone

\section{INTRODUCTION}

The problem of neck pain is common. Especially young adults reported that more than $30 \%$ of population woke with neck pain at least once a week. ${ }^{1}$ Among individuals with neck pain, $37.3 \%$ reported persistent neck pain and related disability and $9.9 \%$ experienced an aggravation during follow-up year with their neck problems. ${ }^{2}$ Telecommunications grow with advancement in scientific technology and changes in industry trends in modern society and the demands for portable devices have increased sharply and further developing mobile technology. Smartphones are becoming increasingly common and have replaced some computer works, and further it has become a culture nowdays. ${ }^{3}$ In Korea, $82.1 \%$ of general population used internet last month in $2013,95 \%$ smartphone users

Received May 20, 2016 Revised Jun 10, 2016

Accepted Jun 13, 2016

Corresponding author Hae-jung Lee

E-mail hlee3652@hanmail.net have internet access in 2012.

Hours of spending on hunched over a smartphone may be damage on person's posture. Visual Display Terminal (VDT) usage could cause consistent posture on the upper quarter of body and musculoskeletal pain in the neck, shoulders, arms and hands. ${ }^{4} \mathrm{Sev}-$ eral studies suggested that neck pain problems result from poor posture, in terms of sustained, long-term, abnormal physiologic loads on the neck. ${ }^{5-7}$ The loads compromise pain-sensitive structures and thereby affect the function of the cervical spine, causing a musculoskeletal imbalance in the upper quarter of the body. For example, a habitual excessively forward head posture has been suggested to be pain provoking, with a consequential reduction in muscle strength. ${ }^{8}$ However, association between physical dimensions of upper quarters of body and the presence of neck pain or dis-

Copylight (C2016 The Korea Society of Physical Therapy

This is an Open Access article distribute under the terms of the Creative Commons Attribution Non-commercial License (Http:// creativecommons.org/license/by-nc/4.o.) which permits unrestricted non-commercial use, distribution, and reproduction in any medium, provided the original work is properly cited. 
comfort have not been clearly established. A few studies could not show any relationship between extreme cervical posture and neck pain. ${ }^{9}$ There is also a lack of association reported between cervical posture and deep cervical flexor endurance. ${ }^{10}$ However, neck muscle endurance was found to be lower for frequent neck pain individuals compared to persons with never or infrequent neck pain. ${ }^{11}$ Only few studies investigated cervical range of motions related to neck pain and a posture, and results of those studies were inconclusive. ${ }^{8,9}$

Functioning in daily activity is considered the most important measure of health problem and it has been suggested that a patient's self-evaluation may be more accurate than the clinical, biomechanical, or physiological indexes. ${ }^{12,13}$ A recent study reported that heavy VDT users who complained frequent neck pain but were not necessarily under health professional services experienced difficulties in daily activities. ${ }^{11}$ There are many studies that musculoskeletal disease and activities of daily living limited due to the use of VDT with computer. However, it has been only few studies reported any association between neck pain and disability in daily activities in smartphone users. Pain in the neck or upper limb is common in smartphone users, and smartphone use is high in the university student population. Accordingly, these high-frequency smartphone users could represent a population. Therefore the purpose of this study was to investigate a relationship between smartphone usage time and neck pain and/or upper limb pain, neck-head posture, range of motions, muscle endurance and disability in university student smartphone users.

\section{METHODS}

\section{Subjects}

Seventy-eight university student volunteers participated in the study. Participants were included if they were older than 18 years of age and had been using a smartphone longer than 1 year. Subjects who had experiences of medical attention for neck and/or related problems within the last 6 months were excluded from participation in the study.

\section{Methods}

\section{1) Procedure}

Physical dimensions of the cervicothoracic spine were measured with the cervical range of movement device (CROM) (Performance
Attainment Associates, St Paul, MN), a stabilizer and a photo for upper quarter of the body, for tests measuring active neck range of motion, neck muscle endurance and head-neck posture respectively. Following all physical testing, background information was obtained by structured interview. This included questions about duration of smartphone usage, recreation and fitness activities, pain and discomfort areas, any recurrent neck pain, and any previous history of neck injury and related treatment. Neck pain and disability data were collected after all measurements using the Korean version of the NDI, WHODAS 2.0-12 items and SFMPQ.

\section{2) Measurement tools}

\section{(1) Head-neck posture by the craniovertebral angle (CVA)}

Head-neck posture was assessed by measuring CVA on a photograph taken in a sagittal craniocervical posture. CVA was calculated between the true horizontal line through the spinous process of $\mathrm{C} 7$ and a line connecting the spinous process of $\mathrm{C} 7$ with the tragus. This angle was chosen as it is considered to be the clinical standard for measuring sagittal craniocervical posture, ${ }^{14}$ which is refers to the degree of forward head posture.

\section{(2) Active range of neck motion by the CROM}

Cervical flexion, extension, rotation and lateral flexion ranges of motion were measured with the CROM device. Subjects were in an upright sitting, looking straight ahead arms resting on their lap, and feet flat on the floor during all range of motion tests. Protraction and retraction of cervical spine were also taken in a position that sagittal rotation was held at 0 using the sagittal inclinometer of the CROM, as described by Lee et al. ${ }^{9}$ The CROM is the most common tool for measuring cervical range of motion. ${ }^{15}$

\section{(3) Endurance of deep neck flexor muscle by the stabilizer}

Neck muscles endurance test was assessed using the stabilizer. Subjects laid hook-lying position on a therapy table with a neutral neck position where their head and neck lined up and straight. The pressure sensor of the stabilizer was placed underneath the neck. Subjects were instructed their head nod gently as if saying 'yes' without using the neck motions which may substitute the sternocleidomastoid muscle so that the pressure sensor measured $2 \mathrm{mmHg}$ above baseline. The testing range was from $20 \mathrm{mmHg}$ to $30 \mathrm{mmHg}$ and the pressure should maintain for the 10 seconds in each $2 \mathrm{mmHg}$ 
without resting. Record the number of times a subject could hold the pressure level. ${ }^{16}$

\section{(4) Neck pain and disability by SFMPQ NDI and WHODAS 2.0}

Neck pain data was collected using SFMPQ. SFMPQ is consisted of the sensory, affective and the present pain intensity as well as overall intensity of pain. Korean version of SFMPQ showed to have good reliability and validity. For functioning data in daily activities, NDI and WHODAS 2.0 were employed in the study. The NDI is a selfreport measure of perceived disability due to neck pain and has been most widely used in different populations. It has 10 items related to daily activities including pain intensity, concentration, work, driving, sleep, recreation, self-care, headache, reading and lifting. The WHODAS 2.0 is generic disability scale that could be used for all persons with health conditions. It is comprised of 12 items with six domains of life, i.e. cognition, mobility, self-care, getting along, life activities and participation. It also includes additional 3 questions regarding effect of difficulties in respondents. WHODAS 2.0 is one of the most widely used functioning measures across different groups and settings. Korean versions of those scales were reported good psychometric properties. ${ }^{1718}$

Table 1. Mean and standard deviation for demographic data with between-group test results

\begin{tabular}{lccc}
\hline Variables & Group 1 & Group 2 & p-value \\
\hline Age (year) & $22.28 \pm 1.88$ & $21.97 \pm 1.35$ & 0.42 \\
Height $(\mathrm{cm})$ & $163.45 \pm 7.25$ & $163.55 \pm 6.55$ & 0.95 \\
Weight $(\mathrm{kg})$ & $56.95 \pm 9.19$ & $56.84 \pm 9.31$ & 0.96 \\
Usage time of & $340.25 \pm 226.28$ & $432.90 \pm 239.72$ & 0.84 \\
Smartphone (minute) & & & \\
\hline
\end{tabular}

Table 2. Mean and standard deviation for between-group test results in posture, neck endurance time and cervical range of motions

\begin{tabular}{lccc}
\hline Variables & Group 1 & Group 2 & p-value \\
\hline Neutral posture $\left(^{\circ}\right)$ & $49.43 \pm 5.37$ & $49.57 \pm 4.53$ & 0.93 \\
Upright posture $\left(^{\circ}\right)$ & $55.85 \pm 5.31$ & $55.50 \pm 4.31$ & 0.75 \\
Endurance $($ second) & $91.65 \pm 43.64$ & $90.87 \pm 45.92$ & 0.94 \\
Flexion $\left(^{\circ}\right)$ & $54.43 \pm 14.04$ & $58.79 \pm 12.67$ & 0.15 \\
Extension $\left(^{\circ}\right)$ & $69.00 \pm 13.53$ & $68.74 \pm 11.03$ & 0.92 \\
Rt. Flexion $\left(^{\circ}\right)$ & $37.10 \pm 8.58$ & $35.37 \pm 8.20$ & 0.37 \\
Lt. Flexion $\left(^{\circ}\right)$ & $40.65 \pm 7.76$ & $38.11 \pm 7.42$ & 0.14 \\
Rt. Rotation $\left(^{\circ}\right)$ & $59.10 \pm 9.88$ & $56.92 \pm 13.74$ & 0.43 \\
Lt. Rotation $\left(^{\circ}\right)$ & $61.30 \pm 10.64$ & $60.37 \pm 11.45$ & 0.71 \\
Protraction $(\mathrm{cm})$ & $3.77 \pm 1.62$ & $3.21 \pm 1.35$ & 0.10 \\
Retraction $(\mathrm{cm})$ & $1.64 \pm 1.37$ & $1.70 \pm 1.24$ & 0.85 \\
\hline
\end{tabular}

Rt, right; Lt, left.

\section{Analysis}

Independent t-test with type I error rate set at 0.05 was used to examine any differences between groups in all variables including pain and disability data. Association between the smartphone usage time and the measured variables was also assessed using Pearson's correlation coefficient. SPSS 21.0 was used for analysis.

\section{RESULTS}

\section{General information}

All 78 subjects were divided into two groups based on neck pain experiences with having neck pain or with no neck pain. Forty subjects had no neck pain reported (group 1) whilst thirty-eight subjects reported neck pain (group 2). The groups did not differ on any of the measured demographic variables. Means, standard deviations, and p-values for the t-tests were presented in Table 1.

\section{Physical function}

All physical measure variables did not show any significantly differences between groups. Results were presented in Table 2.

\section{Neck pain and disability}

Summary of self-report results from the SFMPQ, NDI and WHODAS 2.0 are presented in Figure. 1-1 and 1-2. For the SFMPQ, subjects with neck pain scored significantly higher than subjects with no neck pain in all subscales i.e. sensory, affective, present and usual

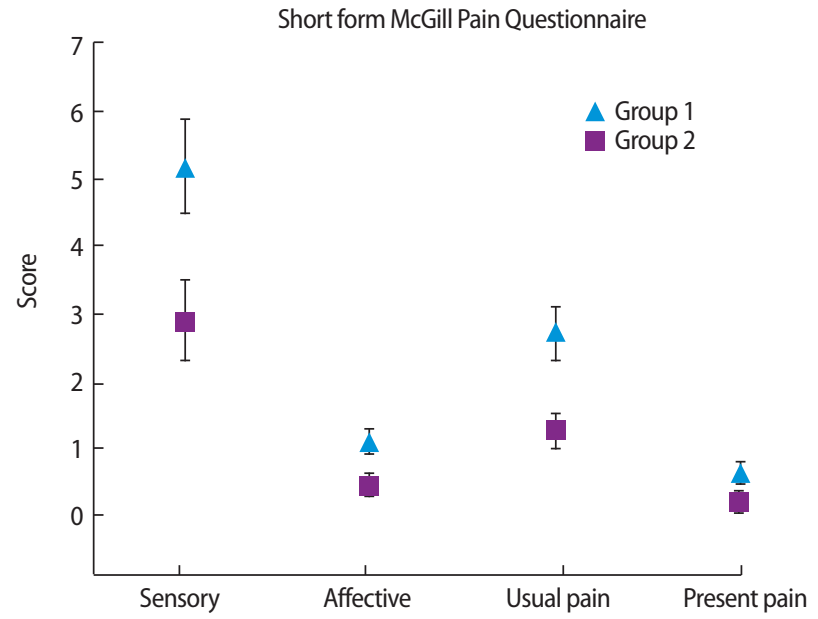

Figure 1-1. Mean scores for the groups on Short Form McGill Pain Questionnaire Subscales: The sensory, affective, usual and present pain. All subscales showed statistically significant at the level of $p<0.05$. 
pain intensity $(\mathrm{p}<0.05)$. Score of NDI in subjects with neck pain had significantly lower than those of no pain subjects. With the

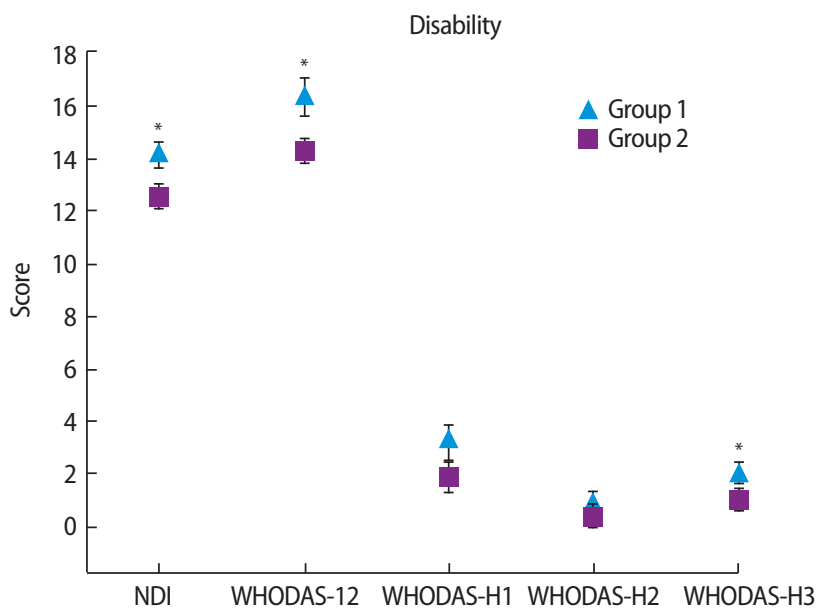

Figure 1-2. Mean scores for the Neck disability index and World Health Organization Disability Assessment Schedule 2.0 Subscales: 12 items in daily activities (12), days of overall difficulties $(\mathrm{H} 1)$ days of totally unable in usual activities $(\mathrm{H} 2)$ and days of reduction in usual activities $(\mathrm{H} 3)$.

*Statistically significant at the level of $p<0.05$.
WHODAS 2.0, on the 12 item of daily activities, subjects with neck pain rated significantly higher than those of no neck pain. On the effect of difficulties, subjects with neck pain counted significantly more days that they consider reduction in usual activities than those with no neck pain, however, the days that subjects were totally unable to carry out activities were not differ between groups.

\section{Correlation}

Correlations were evaluated between measures of all variables and smartphone usage time per day. The relationship between the smartphone usage time and usual pain intensity, neck specific disability score and affected days were positive, indicating that if subjects had longer usage time with their smartphone, they tended to have worse pain in usual $(\mathrm{r}=0.23, \mathrm{p}=0.04)$, more difficult in neck specific activities $(\mathrm{r}=0.24, \mathrm{p}=0.04)$, and perceive more days disturbed in their daily activities $(\mathrm{r}=0.33, \mathrm{p}<0.01)$. Whereas the smartphone usage time was negatively related to left and right side flexion ranges and neck location in neutral sitting posture, which means if

Table 3-1. Correlation between smartphone usage time and physical measure variables $(n=78)$

\begin{tabular}{|c|c|c|c|c|c|c|c|c|c|c|}
\hline & SUT & Flex & Ext & Rt SF & Lt SF & Rt Rot & Lt Rot & Pro-Ret & Neck End & Sit- post \\
\hline SUT & 1 & -0.10 & -0.10 & $-0.25^{*}$ & $-0.29^{\star}$ & -0.06 & 0.02 & $-0.22^{*}$ & -0.13 & $-0.33^{*}$ \\
\hline Flex & & 1 & 0.19 & $0.27^{\star}$ & $0.27^{*}$ & $0.26^{*}$ & $0.28^{*}$ & -0.01 & -0.01 & $0.23^{*}$ \\
\hline Ext & & & 1 & $0.44^{*}$ & $0.50^{*}$ & $0.39^{*}$ & $0.44^{*}$ & -0.06 & 0.07 & -0.07 \\
\hline Rt SF & & & & 1 & $0.76^{*}$ & $0.44^{*}$ & $0.50^{*}$ & 0.12 & $0.30^{*}$ & 0.05 \\
\hline Lt SF & & & & & 1 & $0.45^{\star}$ & $0.50^{*}$ & 0.14 & 0.20 & 0.16 \\
\hline Rt Rot & & & & & & 1 & $0.60^{*}$ & 0.06 & $0.26^{*}$ & -0.03 \\
\hline Lt Rot & & & & & & & 1 & 0.14 & 0.08 & -0.07 \\
\hline Pro-Ret & & & & & & & & 1 & -0.02 & $0.24^{*}$ \\
\hline Neck End & & & & & & & & & 1 & -0.07 \\
\hline Sit- post & & & & & & & & & & 1 \\
\hline
\end{tabular}

SUT: smartphone usage time, Flex: flexion, Ext: extension, Rt SF: right side flexion, Lt SF: left side flexion, Rt Rot: right rotation, Lt Rot: left rotation, Pro-Ret: protractionretraction, Neck End: neck flexor endurance, Sit-post: sitting posture. ${ }^{*} \mathrm{p}<0.05$.

Table 3-2. Correlation between smartphone usage time and self-report variables $(n=78)$

\begin{tabular}{lcccccccc}
\hline & SUT & SFMPQ1 & SFMPQ2 & SFMPQ3 & SFMPQ4 & NDI & WHODAS-12 & WHODAS-H \\
\hline SUT & 1 & 0.10 & 0.11 & $0.23^{*}$ & $0.21^{*}$ & $0.24^{*}$ & 0.06 & $0.32^{*}$ \\
SFMPQ1 & & 1 & $0.66^{*}$ & $0.62^{*}$ & $0.41^{*}$ & $0.43^{*}$ & 0.07 & 0.12 \\
SFMPQ2 & & 1 & $0.46^{*}$ & 0.11 & $0.21^{*}$ & 0.14 & 0.11 \\
SFMPQ3 & & & 1 & $0.53^{*}$ & $0.47^{*}$ & 0.13 & $0.24^{*}$ \\
SFMPQ4 & & & & 1 & $0.48^{*}$ & 0.09 & $0.29^{*}$ \\
NDI & & & & & & & $0.35^{*}$ & $0.23^{*}$ \\
WHODAS-12 & & & & & & & 1 & $0.62^{*}$ \\
WHODAS-H & & & & & & & \\
\hline
\end{tabular}

SUT: smartphone usage time, SFMPQ: short form McGill pain questionnaire, NDI: neck disability index, WHODAS: world health organization disability assessment schedule ${ }^{*} p<0.05$. 
subjects spent longer time on a smartphone, they had limited in right and left flexion ranges $(r=-0.25, p=0.03$, and $r=-0.29, p=0.01$, respectively) and tended to sit upright $(\mathrm{r}=0.33, \mathrm{p}<0.01)$. The results were summarized in Table 3-1 and 3-2.

\section{DISCUSSION}

In the current study, participants used their smartphone every day and most of them spent more than two hours per day on using it. These high-frequency smartphone users were identified themselves as heavy smartphone users. Participants with neck pain and participants with no neck pain demonstrated similar physical measures including their smartphone usage time but differed with respect to their perceived pain intensity and disability questionnaire responses. It was found that functioning in daily activity was the most affected aspect for heavy smartphone users.

In the functioning of daily activities, subjects with neck pain scored higher than those of no neck pain group whether activities were specifically related to neck or not. Further persons with neck pain tended to have more days to be perceived as disturbed in their daily routines than those with no neck pain. The responses on the disability questionnaires were similar to the rated intensity of their usual pain. These data suggest that when heavy smartphone users experience neck pain, they become more distressed and aware of their pain. On the SFMPQ, neck pain subjects scored higher than those with no neck pain on all subscales. Subjects who had neck pain rated their pain not only as more aching, throbbing, stabbing, shooting but also as more frightening, punishing or sickening than those without neck pain. This result found that subjects in the study, heavy smartphone users with neck pain, showed similar behavior to those with clinical neck pain population.11 Subjects with neck pain perceived their usual pain more intensively in general than those with no pain in the neck. These results were consistent with previous studies, which reported subjects with frequent neck pain reported more severe pain than those with no or less frequent pain. ${ }^{19}$ This aspect of pain response can be seen as consistent with clinical neck pain studies, where a high level of psychological distress is correlated with being more likely to use health care services. ${ }^{20}$ Therefore, it may imply that heavy smartphone users with neck pain can be examined as early as possible and put them on early intervention accordingly.
Interestingly, any of physical dimensions in the study was not found to be different between subjects with neck pain and subjects without neck pain. The observed ranges of motions in cervical spine in the study were consistent with previous studies' results. Lee et al. ${ }^{19}$ reported that heavy computer users with neck pain had sensitization effect on the 2 nd measurement, which is reduced ranges of motion at the second occasion of measurement. These sensitized ranges were consistent with the current study results in flexion, side flexions, rotations, protraction and retraction ranges. ${ }^{21}$ Neck muscle endurance was also found to be low in all subjects with or without their neck pain. Subjects tended to cease the test due to muscle fatigue and/or due to pain. Significant reduction of neck muscle strength has been reported in subjects with neck pain. It seems that impairments of neck range of motions and neck muscle function may develop due to heavy usage of a smartphone.

There was a significant negative relationship between the time of smartphone use and neck side flexions. The size of the smartphone is relatively small when comparing to the computer VDT. The posture people adopt as they look at their smartphone increases the stress on the neck. People are using their smartphone not only sitting and standing but also lying on prone and side lying. Further, people are using their smartphone with one hand often with titled head due to the size of the equipment. It may cause excessive stress on the related structures that the more use a smartphone, the less side flexion movements could occur. It may protect against stress on those structures and decrease discomfort. It was also found that if subjects had spent more time on their smartphone, subjects sit more upright. It is possible that upright posture could make less stress on pain sensitive structures on the cervical spine and may protect against such stress. Therefore, it can be suggested that stretching exercise have a role in neck pain rehabilitation for heavy smartphone users.

In conclusion, comparing groups with and without neck pain in heavy smartphone users, higher score in pain intensity of SFMPQ and both disability questionnaires, NDI and WHODAS 2.0, for the group with neck pain were observed, but no physical dimensions in neck range of motions, muscle endurance and head-neck posture were different across groups. Rather, their observed physical dimensions in subjects found to be similar to those in clinical neck pain population. These data suggested that the pain and functioning in daily activities are useful measurements to distinguish between groups with and without neck pain. Longitudinal study of these 
heavy smartphone users with neck pain is needed.

\section{REFERENCES}

1. Gordon SJ, Trott P, Grimmer KA. Waking cervical pain and stiffness, headache, scapular or arm pain: Gender and age effects. J Physiother. 2002;48(1):9-15.

2. Cote P, Cassidy JD, Carroll LJ et al. The annual incidence and course of neck pain in the general population: A population-based cohort study. Pain. 2004;112(3):267-73.

3. Seo E. Changes in accommodative function after VDT work. J Kor Oph Opt Soc. 2012;17(3):285-91.

4. Saito S, Miyao M, Kondo T et al. Ergonomic evaluation of working posture of VDT operation using personal computer with flat panel display. Ind Health. 1997;35(2):264-70.

5. Kwon JW, Nam KS, Choi YW et al. The effect of different head positions in sitting on head/shoulder posture and muscle activity. J Kor Phys Ther. 2013;25(4):217-23.

6. Nam KS, Kwon JW. The effects of head position in different sitting postures on muscle activity with/without forward head and rounded shoulder. J Kor Phys Ther. 2014;26(3):140-6.

7. Grimmer-Somers K, Milanese S, Louw Q. Measurement of cervical posture in the sagittal plane. J Manip Physiol Ther. 2008;31(7):509-17.

8. Hanten WP, Olson SL, Russell JL et al. Total head excursion and resting head posture: Normal and patient comparisons. Arch Phys Med Rehab. 2000;81(1):62-6.

9. Lee H, Nicholson LL, Adams RD. Cervical range of motion associations with subclinical neck pain. Spine. 2003;29(1):33-40.

10. Grimmer K, Trott $P$. The association between cervical excursion angles and cervical short flexor muscle endurance. J Physiother. 1998;44(3): 201-7.
11. Lee H, Nicholson LL, Adams RD. Neck muscle endurance, self-report, and range of motion data from subjects with treated and untreated neck pain. J Manip Physiol Ther. 2005;28(1):25-32.

12. Kim K, Kim EK, Lee DK. Effects of pnf patterns exercise on pain, functional disability and fear avoidance belief in chronic low back pain patients. J Kor Phys Ther. 2014;26(2):110-6.

13. Lee H, Song J. An overview of the icf s use in Korea. J Kor Phys Ther. 2015; 27(5):356-63.

14. Gadotti IC, Magee D. Validity of surface markers placement on the cervical spine for craniocervical posture assessment. Man Ther. 2013;18(3): 243-7.

15. Audette I, Dumas J-P, Côté JN et al. Validity and between-day reliability of the cervical range of motion (crom) device. J Orthop Sport Phys Ther. 2010;40(5):318-23.

16. Jull GA, O'Leary SP, Falla DL. Clinical assessment of the deep cervical flexor muscles: The craniocervical flexion test. J Manip Physiol Ther. 2008;31(7):525-33.

17. Lee H, Nicholson LL, Adams RD et al. Development and psychometric testing of Korean language versions of 4 neck pain and disability questionnaires. Spine. 2006;31(16):1841-5.

18. Lee HJ, Kim DJ. Internal consistency and concurrent validity of Korean language version of WHODAS 2.0: 12 item-self administered. J Kor Phys Ther. 2011;23(6):23-9.

19. Lee H, Nicholson LL, Adams RD et al. Proprioception and rotation range sensitization associated with subclinical neck pain. Spine. 2005; 30(3):E60-7.

20. Leclerc A, Niedhammer I, Landre MF et al. One-year predictive factors for various aspects of neck disorders. Spine. 1999;24(14):1455-62.

21. Lee H, Nicholoson LL, Adams RD et al. Body chart pain location and side-specific physical impairment in subclinical neck pain. J Manip Physiol Ther. 2005;28(7):479-86. 